\title{
Bäcklund transformation, auto-Bäcklund transformation and exact solutions for KdV-type equations
}

\author{
O. H. El-Kalaawy \\ Department of Mathematics, Faculty of Science, Beni-Suef University, Beni-Suef 62511, Egypt \\ Email: ohkalaawy7@gmail.com
}

Copyright (C)2014 EL-Kalaawy. This is an open access article distributed under the Creative Commons Attribution License, which permits unrestricted use, distribution, and reproduction in any medium, provided the original work is properly cited.

\begin{abstract}
In plasma physics, fluid dynamics and nonlinear optics, Korteweg-de Vries (KdV)-type equations are used to describe certain phenomena (ion acoustic wave in plasma, quantum hydrodynamic model, wave motion on the surface of shallow water and the unidirectional propagation of long wave of small amplitude and exists in many physical branches). In this paper, KdV-type equations are investigated. We are used Bäcklund Transformation to obtain new exact solutions for the (KdV)-type equations. The method of characteristics is used and the Bäcklund transformation are employed to generate new solutions from the old ones. By the homogenous balance method, we derive an auto-Bäcklund Transformation (ABT) for the KdV equation. Thus, families of solution for KdV-type equations are obtained.
\end{abstract}

Keywords:KdV-type equations, Bäcklund transformation, auto-Bäcklund transformation and exact solutions.

\section{Introduction}

Nonlinear evolution equations (NLEEs) are important mathematical models to describe physical phenomena. They are also an important field in the contemporary study of nonlinear physics, especially in soliton theory. The research on the explicit solution and integrability in helpful in clarifying the movement of matter under nonlinear interaction and plays an important role in scientifically explaining the physical phenomena see for example, fluid mechanics, plasma physics, quantum hydrodynamic model, optical fibers, solid state physics, chemical kinematic, chemical physics and geochemistry. In this paper we will consider the following KdV-type equations.

The KdV equation

$$
q_{t}+6 q_{x}+6 q q_{x}+q_{x x x}=0
$$

The MKdV equation in $q_{x}$

$$
q_{t x}+\left(q_{x}\right)^{2} q_{x x}+q_{x x x x}=0
$$

The investigation of the exact solutions of nonlinear partial differential equations (NPDEs) plays an important role in the study of nonlinear physical phenomena. In the past decades, there has been significant progression in the development of methods such as the inverse scattering method [1-2], Hirotas bilinear method [3], Painlevé 
expansions [4-7], truncated Painlevé [8], homogeneous balance method [9-10], the linearized transformation method [11], tanh function method [12-15] and several ansatz method [16-17]. The Bäcklund transformation (BT) technique is one of the direct methods for generating a new solution of NLEEs from a known solution of that equation (see, for example, [18-20]). Previously, Konno and Wadati [21], for example, had derived some BTs for the NEEs of the AKNS class [2,21]. These BTs explicitly express the new solutions in terms of the known solutions of the NLEEs and the corresponding wave functions, which are solutions of the associated AKNS system. The AKNS system is a linear eigenvalue problem in the form of a system of first-order partial differential equations. Therefore, the problem of obtaining new solutions by BTs is equivalent to obtaining the wave function. The main aim of this paper is to derive BT, ABT and construct solutions for the KdV-type equations. [1].

\section{The AKNS system and the BT for the NLEEs}

It is well known that many integrable NLEEs can be derived from the following AKNS system eigenvalue problem, defined in the from

$\psi_{x}=U \psi, \psi_{t}=V \psi$

$\psi=\left(\psi_{1}, \psi_{2}\right)^{T}, U=\left(\begin{array}{ll}\eta & q \\ r & -\eta\end{array}\right), V=\left(\begin{array}{ll}A & B \\ C & -A\end{array}\right)$

where $\eta$ is a parameter, independent of $\mathrm{x}$ and $\mathrm{t}, \mathrm{q}$ and $\mathrm{r}$ are function of $\mathrm{x}$ and $\mathrm{t}$, while A,B and $\mathrm{C}$ are scalar function of $q(x, t)$. The integrability condition $\psi_{x t}=\psi_{t x}$ yields at once the evolution equations in the general form

$$
-A_{x}+q C-r B=0, \quad q_{t}-B_{x}-2 q A+2 \eta B=0, \quad r_{t}-C_{x}-2 \eta C+2 r A=0 .
$$

By suitably choosing A, B, C and $\mathrm{r}$ in (5), we obtain various NLEEs which $q$ must satisfy. Konno and Wadati [21] introduced the relation

$\Gamma=\frac{\psi_{1}}{\psi_{2}}$

and for any of the NLEEs derived a BT with the following form:

$q^{\prime}=q+F(\Gamma, \eta)$

where $q^{\prime}$ is the new solution generated from the old $q$.

\subsection{The $\mathrm{KdV}$ equation}

$r=-1$

$A=-4 \eta^{3}-2 \eta q-q_{x}-6 \eta, \quad B=-4 \eta^{2} q-6 q-2 q^{2}-2 \eta q_{x}-q_{x x}, \quad C=4 \eta^{2}+2 q+6$.

To derive the new solution $q^{\prime}$ from the known solution $q$ we use the BT

$q^{\prime}=q-2 \frac{\partial \Gamma(x, t)}{\partial x}$

\subsection{For the MKdV equation}

$$
\begin{aligned}
& r=-q=\frac{u_{x}}{2} \\
& A=-4 \eta^{3}-\frac{1}{2} \eta\left(u_{x}\right)^{2}, \quad B=\frac{1}{2} u_{x x x}+\eta u_{x x}+2 \eta^{2} u_{x}+\frac{1}{4} u_{x}^{3}, \quad C=-\frac{1}{2} u_{x x x}+\eta u_{x x}-2 \eta^{2} u_{x}-\frac{1}{4} u_{x}^{3} .
\end{aligned}
$$

To derive the new solution $q^{\prime}$ from the known solution $q$ we use the BT

$q^{\prime}=q+4 \tan ^{-1}(\Gamma)$. 


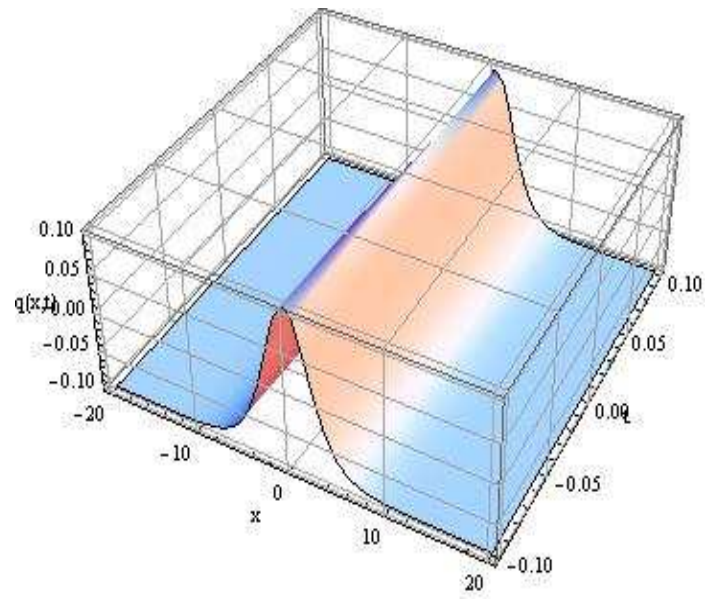

Figure 1: (a)

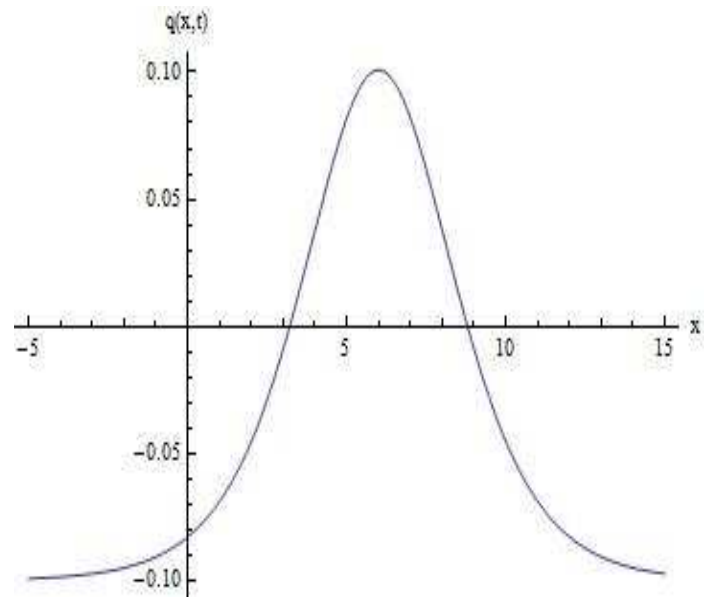

The solition solution of (15) for different values $\eta=.2, c=5$ and $q_{0}=-0.1$.

Proposition. The MKdV equation (2) is invariant under the BT

$$
\begin{aligned}
& \frac{\partial u^{\prime}}{\partial x}+\frac{\partial u}{\partial x}=4 \eta \sin \left(\frac{u^{\prime}-u}{2}\right) \\
& \frac{\partial u^{\prime}}{\partial t}-\frac{\partial u}{\partial t}=4 A \sin \left(\frac{u^{\prime}-u}{2}\right)+2(B-C)+2(B+C) \cos \left(\frac{u^{\prime}-u}{2}\right)
\end{aligned}
$$

where $u$ is a solution of the equation, $u^{\prime}$ also satisfy the same equation and the BT for the class of the AKNS system can be constructed from the inverse scattering formalism (Proof, see Appendix A).

\section{The known solution is a constant}

\subsection{The $\mathrm{KdV}$ equation}

To construct the solution of equation (1), we start with the zero solutions $q(0)=q_{0}$. By substituting this solution in the two matrices $\mathrm{U}$ and $\mathrm{V}$ we obtain:

$$
U=\left(\begin{array}{ll}
\eta & q_{0} \\
-1 & -\eta
\end{array}\right), V=\left(\begin{array}{lc}
-4 \eta^{3}-2 \eta q_{0}-6 \eta & -4 \eta^{2} q_{0}-6 q_{0}-2 q_{0}^{2} \\
4 \eta^{2}+2 q_{0}+6 & 4 \eta^{3}+2 \eta q_{0}+6 \eta
\end{array}\right)
$$

From the AKNS system, we get the solution

$\psi=\psi_{0} e^{U \rho}=\psi_{0}\left[I+U \rho+\frac{U^{2} \rho^{2}}{2 !}+\frac{U^{3} \rho^{3}}{3 !}+\frac{U^{4} \rho^{4}}{4 !}+\ldots\right]=\psi_{0}\left(\begin{array}{ll}\cosh \alpha \rho+\frac{\eta}{\alpha} \sinh \alpha \rho & \frac{q_{0}}{\alpha} \sinh \alpha \rho \\ \sinh \rho & \cosh \alpha \rho-\frac{\eta}{\alpha} \sinh \alpha \rho\end{array}\right)$,

where $\alpha^{2}=\eta^{2}-q_{0}$. Then the solution of $\mathrm{KdV}$ equation corresponding to the known solution $q(0)=q_{0}$ anther $\psi_{0}=(0,1)^{T}$,

$$
q^{\prime}=\frac{q_{0}\left(-\eta^{2}-3 \alpha^{2}+\left(\alpha^{2}+\eta^{2}\right) \cosh 2 \alpha \rho-2 \eta \alpha \sinh 2 \alpha \rho\right)}{2(\alpha \cosh \alpha \rho-\eta \sinh \alpha \rho)^{2}},
$$

where $\rho=x-\left(4 \eta^{2}+2 q_{0}+6\right) t$, and $\alpha^{2}=\eta^{2}-q_{0}$. We have represented this solution for a set of parameter values in Fig. 1. 


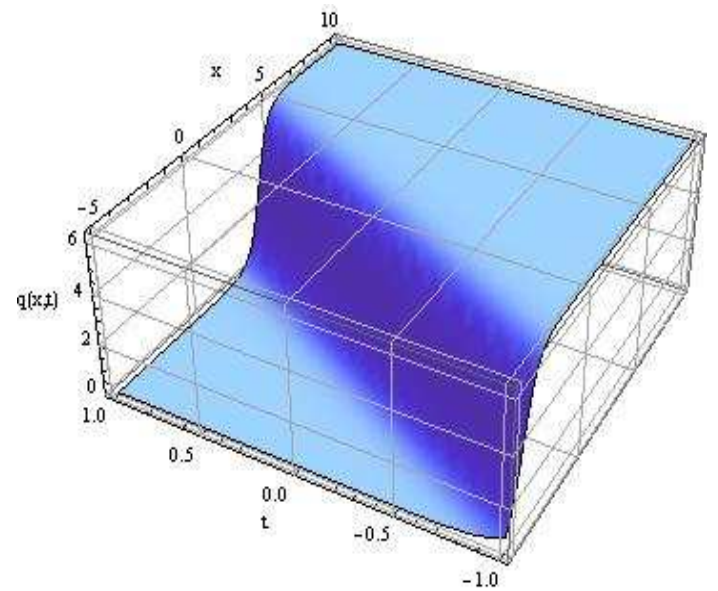

Figure 2: (a)

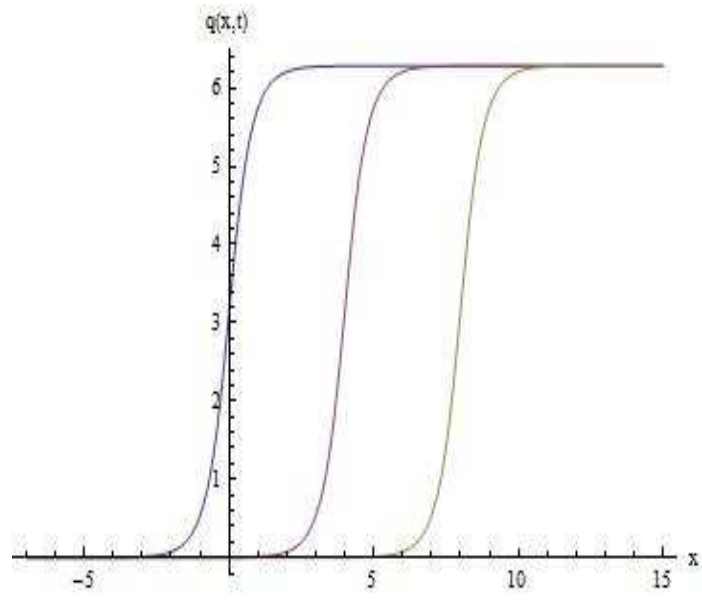

(b)

The kink solution of (19) for different values $\eta=1$ and $c=1$. (a)in the 3D and (b) in the plane at $t=0,1,2$.

\subsection{The $\mathrm{MKdV}$ equation}

To construct the solution of equation (2), we start with the zero solutions $q(0)=0$. By substituting this trivial solution in the two matrices $\mathrm{U}$ and $\mathrm{V}$ we obtain:

$U=\left(\begin{array}{ll}\eta & 0 \\ 0 & -\eta\end{array}\right), V=\left(\begin{array}{ll}-4 \eta^{3} & 0 \\ 0 & 4 \eta^{3}\end{array}\right)$

From the AKNS system (3)-(4)

$d \psi=U \psi d x+V \psi d t=(U d x+V d t) \psi$

and thus we get

$$
d \psi=U \psi d \rho, d \rho=d x-\left(4 \eta^{2}\right) d t, \quad \rho=x-\left(4 \eta^{2} t\right)
$$

Then the solution of (23) is

$\psi=\psi_{0}\left(\begin{array}{ll}e^{\eta \rho} & 0 \\ 0 & e^{-\eta \rho}\end{array}\right)$

Choosing $\psi_{0}=(1,1)^{T}$, we obtain the new solution of the MKdV equation corresponding to the known solution $q(0)=0$

$q^{\prime}=4 \tan ^{-1}\left(e^{2 \eta\left(x-4 \eta^{2} t\right)}\right)$.

We have represented this solution for a set of parameter values in Fig. 2.

\section{The known solution $q=q(x, t)$ is a simple function}

In this case the system (3)-(4) cannot be solved for the vector $\Psi$ as a whole, but it can be solved in the component $\psi_{1}$ and $\psi_{2}$ separately. From (3)-(4), after inserting the known solution $q(x, t)$ of the KdV equation into the corresponding matrices $\mathrm{U}$ and $\mathrm{V}$ we will have the following system of partial differential equations for unknowns $\psi_{1}$ and $\psi_{2}$ :

$$
\begin{gathered}
\psi_{1 x}=\eta \psi_{1}+q \psi_{2} \\
\psi_{2 x}=r \psi_{1}-\eta \psi_{2} \\
\psi_{1 t}=A \psi_{1}+B \psi_{2} \\
\psi_{2 t}=C \psi_{1}-A \psi_{2}
\end{gathered}
$$


These equations are compatible under the conditions of the assumed values of the matrices $\mathrm{U}$ and $\mathrm{V}$ connected with the KdV equation under consideration. Solving for $\psi_{1}$ from (20b) yields

$\psi_{1}=\frac{1}{r}\left(\psi_{2 x}-\eta \psi_{2}\right)$

Substituting this $\psi_{1}$ into (20d) we have

$$
C \psi_{2 x}-r \psi_{2 t}=\frac{1}{2}\left(C_{x}-r_{t}\right) \psi_{2}
$$

This is linear first-order partial differential equation with $\psi_{2}$ as the unknown function; it can be solved by the method of characteristics or another method. After $\psi_{2}$ has been obtained from (22), and substituting it into (21), we will obtain $\psi_{1}$. To be more specific, we apply the last technique in the following example:

\subsection{The KdV equation}

Let

$q(x, t)=\frac{x-6 t}{3(2 t+1)}, \quad t \neq-\frac{1}{2}$.

By direct calculation one check that equation (23) is a solution of the KdV equation (1). Inserting equation (23) into equation (22), together with equations (3) and (4), gives

$$
\left(4 \eta^{2}+\frac{2(x-6 t)}{3(2 t+1)}+6\right) \psi_{2 x}+\psi_{2 t}=\frac{\psi_{2}}{3(2 t+1)} .
$$

equation (24) has the following system ODEs as its characteristic equations:

$\frac{d x}{d t}=\left(4 \eta^{2}+6\right)+\frac{2(x-6 t)}{3(2 t+1)}$,

$\frac{d \psi_{2}}{\psi_{2}}=\frac{d t}{3(2 t+1)}$.

Solving these two equations gives the general solution of the unknown $\psi_{2}$ in equation (24), which reads:

$$
\begin{array}{r}
\psi_{2}=(2 t+1)^{1 / 6} F(\xi) \\
\xi=(x+3)(2 t+1)^{-1 / 3}-3\left(\eta^{2}+1\right)(2 t+1)^{2 / 3},
\end{array}
$$

where $F$ is an arbitrary function. Substituting equations (23) and (27) into equation (21) gives the general solution of $\psi_{1}$, which reads:

$$
\psi_{1}=-(2 t+1)^{-1 / 6} F^{\prime}(\xi)-\eta(2 t+1)^{1 / 6} F(\xi) .
$$

To determine the function $F(\xi)$, we substitute from equations (23), (27) and (28) into equation (20a), then $F(\xi)$ must satisfy the following Airy equation [22]

$$
\frac{d^{2} F(\xi)}{d \xi^{2}}+\frac{\xi}{3} F(\xi)=0
$$

Therefore, we obtain the function $F(\xi)$ as follows:

$$
F(\xi)=c_{1} A i(\xi)+c_{2} B i(\xi),
$$

where $A i(\xi)$ and $B i(\xi)$ are two Airy functions [22], while $c_{1}$ and $c_{2}$ are two arbitrary constants After $F(\xi)$ has been determined, equations (27), (28) and (6) leads to

$$
\Gamma=-(2 t+1)^{-1 / 3} \frac{F^{\prime}(\xi)}{F(\xi)}-\eta,
$$

then substituting this $\Gamma$ and equation (23) into the BT (7) yields the new solution $q^{\prime}$ of the KdV equation (1) corresponding to the known solution (23)

$$
q^{\prime}(x, t)=\frac{x-6 t}{3(2 t+1)}+2(2 t+1)^{-2 / 3} \frac{d}{d \xi}\left(\frac{F^{\prime}(\xi)}{F(\xi)}\right) .
$$




\section{The known solution is a traveling wave}

In this we suppose that the components $q$ and $r$ of the all matrix $U$ are function of $\rho$

$q=q(\rho), \quad r=r(\rho)$, where $\rho=x-K t$.

then the components $A, B$ and $C$ of the matrix $V$ determined by equations (5) are also functions of $\rho$ :

$$
A=A(\rho), \quad B=B(\rho), \quad \text { and } \quad C=C(\rho) .
$$

We require the quantity

$\beta=(A+K \eta)^{2}+(B+K q)(C+K r)$,

to be constant with respect to $\rho$ (or $x$ and $t$ ). Under these assumptions, the following result holds, which is crucial in the subsequent exact solution. Solving system (22) by applying the method of characteristics as section 4, the PDE (22) possesses the following characteristic equations:

$\frac{d t}{-r}=\frac{d x}{C}=\frac{2 d \psi_{2}}{\left(C_{x}-r_{t}\right) \psi_{2}}$.

Using equations (33) and (34) substituting in equation (36) gives

$\frac{d t}{-r}=\frac{d \rho}{(C+K r)}=\frac{2 d \psi_{2}}{(C+K r)_{\rho}^{\prime} \psi_{2}}$.

These equations yield the following system of ODEs

$\frac{d\left(\ln \psi_{2}\right)}{d \rho}=\frac{(C+K r)_{\rho}^{\prime}}{(2(C+K r))}$

$\frac{d \rho}{d t}=-\frac{(C+K r)}{r}$

Integrating (39) we get

$$
-t+k_{1}=\int \frac{r d \rho}{(C+K r)}
$$

where $k_{1}$ is an integration constant. Integrating equation (38) leads to

$\psi_{2}=k_{2}(C+K r)^{1 / 2}$,

where $k_{2}$ is another integration constant.

Denoting

$$
\sigma(\rho)=\int \frac{r d \rho}{(C+K r)} .
$$

Substituting from equation (42) into equation (40), we have

$$
\sigma(\rho)+t=k_{1} .
$$

From equations (41) and (43), we obtain the general solution of equation (22):

$\psi_{2}=(C+K r)^{1 / 2} F(\xi)$,

where

$\xi=\sigma(\rho)+t$ 
and $F(\xi)$ is a differential function of $\xi$. Substituting equation (44) into equation (21) gives the general solution for $\psi_{1}$ :

$\psi_{1}=(C+K r)^{-1 / 2}\left(F_{\xi}^{\prime}+(A+K \eta) F\right)$.

To determine the function $F(\xi)$, we substitute equations (44) and (46) into equation (20a), and we find that $F(\xi)$ must satisfy the following second order ODE:

$F_{\xi \xi}^{\prime \prime}-\beta F=0$,

where $\beta$ is a constant defined in equation (35). According to the sign of $\beta$, equation (47) will have the following three different solutions:

$$
\begin{array}{r}
F(\xi)=c_{1} \xi+c_{2} \quad \text { when } \quad \beta=0, \\
F(\xi)=c_{1} \sinh \omega\left(\xi+c_{2}\right) \quad \text { when } \quad \beta>0 \quad \omega^{2}=\beta, \\
F(\xi)=c_{1} \sin \omega\left(\xi+c_{2}\right) \quad \text { when } \beta<0 \quad \omega^{2}=-\beta,
\end{array}
$$

where $c_{1}$ and $c_{2}$ are integration constants. Substituting these solutions into equations (46) and (44), respectively, we obtain the corresponding different solution of system (3)-(4):

$$
\begin{aligned}
& \left(\begin{array}{l}
\psi_{1} \\
\psi_{2}
\end{array}\right)=\left(\begin{array}{c}
(C+K r)^{-1 / 2}\left[(A+K \eta)\left(c_{1} \xi+c_{2}\right)+c_{2}\right] \\
(C+K r)^{1 / 2}\left(c_{1} \xi+c_{2}\right)
\end{array}\right), \text { when } \beta=0, \\
& \left(\begin{array}{c}
\psi_{1} \\
\psi_{2}
\end{array}\right)=\left(\begin{array}{c}
c_{1}(C+K r)^{-1 / 2}\left[(A+K \eta) \sinh \omega\left(c_{2}+\xi\right)+\omega \cosh \omega\left(\xi+c_{2}\right)\right] \\
c_{1}(C+K r)^{1 / 2} \sinh \omega\left(\xi+c_{2}\right)
\end{array}\right), \text { when } \beta>0, \\
& \left(\begin{array}{c}
\psi_{1} \\
\psi_{2}
\end{array}\right)=\left(\begin{array}{c}
c_{1}(C+K r)^{-1 / 2}\left[(A+K \eta) \sin \omega\left(c_{2}+\xi\right)+\omega \cos \omega\left(\xi+c_{2}\right)\right] \\
c_{1}(C+K r)^{1 / 2} \sin \omega\left(\xi+c_{2}\right)
\end{array}\right), \text { when } \beta<0 .
\end{aligned}
$$

These results (49a)-(49c) are valid for any NLEE contained in the AKNS system (3)-(4), provided they satisfy assumption (33) and (34).

Now, applying the results obtained here and the known traveling wave solution of the KdV-type equations obtained in section 3. allows us to construct a new class of solutions of the corresponding KdV-type equations by means of the BTs. The constant $\beta$ defined by (35) is zero and, therefore, the corresponding solution of the AKNS system (3)-(4) is (49a). By substituting equation (49a) into equation (6) we get the common expression of $\Gamma$ for the KdV-type equations.

$\Gamma=(C+K r)^{-1}\left[(A+K \eta)+\left(\frac{1}{c+\xi}\right)\right], \quad c=c_{1} / c_{2}$.

\subsection{The KdV equation}

Let $\quad q=\frac{q_{0}\left(-\eta^{2}-3 \alpha^{2}+\left(\alpha^{2}+\eta^{2}\right) \cosh 2 \alpha \rho-2 \eta \alpha \sinh 2 \alpha \rho\right)}{2(\alpha \cosh \alpha \rho-\eta \sinh \alpha \rho)^{2}}$,

where $\rho=x-\left(4 \eta^{2}+2 q_{0}+6\right) t$, and $\alpha^{2}=\eta^{2}-q_{0}$. Clearly (51) satisfies the KdV equation (1) as can be seen by direct substitution. Substituting in equation (35) we obtain that the constant $\beta$ is equal zero. Substituting in two relations (43) and (45)

$$
\xi=\int \frac{r d \rho}{(C+K r)}+t=\frac{1}{16 \alpha^{2} q_{0}}\left(-2 \eta \cosh 2 \alpha \rho+\frac{\left(2 \eta^{2}-q_{0}\right)}{\alpha} \sinh 2 \alpha \rho-2 q_{0} \rho+16 \alpha^{2} q_{0} t\right) .
$$

Substituting in relation (50), then

$$
\Gamma=\frac{-2 \eta^{2} \sinh \alpha \rho+2 \eta \alpha \cosh \alpha \rho+q_{0} \sinh \alpha \rho}{\eta \sinh \alpha \rho-\alpha \cosh \alpha \rho}-\frac{1}{4 \alpha^{2} q_{0}}\left(\frac{1}{c+\xi}\right)(\eta \sinh \alpha \rho-\alpha \cosh \alpha \rho)^{2},
$$




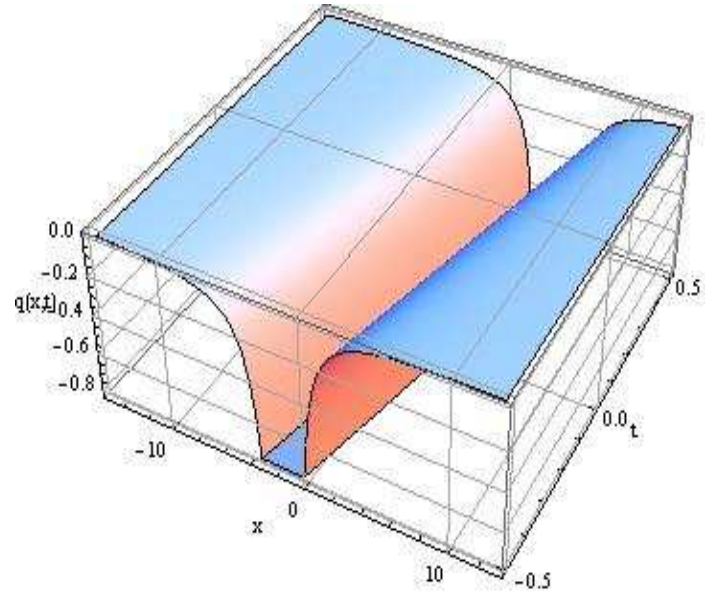

Figure 3: (a)

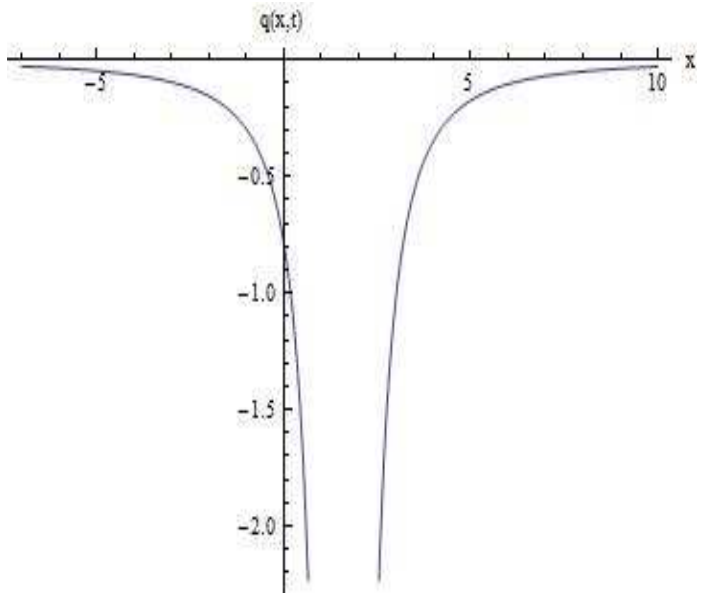

The singular solition solution of (54) for different values $\eta=.2, c=5$ and $q_{0}=-0.1$.

Then substituting from relations (53) and (51) into (7), we obtain

$q^{\prime}=\frac{q_{0}\left(-\eta^{2}-3 \alpha^{2}+\left(\alpha^{2}+\eta^{2}\right) \cosh 2 \alpha \rho-2 \eta \alpha \sinh 2 \alpha \rho\right)}{2(\alpha \cosh \alpha \rho-\eta \sinh \alpha \rho)^{2}}$

$-2\left(\frac{-2 \eta^{2} \sinh \alpha \rho+2 \eta \alpha \cosh \alpha \rho+q_{0} \sinh \alpha \rho}{\eta \sinh \alpha \rho-\alpha \cosh \alpha \rho}-\frac{1}{4 \alpha^{2} q_{0}}\left(\frac{1}{c+\xi}\right)(\eta \sinh \alpha \rho-\alpha \cosh \alpha \rho)^{2}\right){ }_{x}$.

We have represented this solution for a set of parameter values in Fig. 3.

\subsection{The MKdV equation}

Let $\quad q=4 \tan ^{-1}\left(e^{2 \eta \rho}\right)$.

Clearly (55) satisfies the KdV equation (2) as can be seen by direct substitution. Substituting in equation (35) we obtain that the constant $\beta$ is equal zero. Substituting in two relations (43) and (45)

$$
\xi=\int \frac{r d \rho}{(C+K r)}+t=\frac{1}{32 \eta^{3}}\left(32 \eta^{3} t+e^{-4 \eta \rho}-4 \eta \rho\right)
$$

Substituting in relation $(56)$, then

$\Gamma=-\frac{e^{2 \eta \rho}\left(2+e^{4 \eta \rho}-32 \eta^{3}(t+c)+4 \eta \rho\right)}{1+4 \eta e^{4 \eta \rho}\left(8 \eta^{2}(t+c)-\rho\right)}$,

Then substituting from relations (57) and (55) into (11), we obtain

$q^{\prime}=4\left(\tan ^{-1}\left(e^{2 \eta \rho}\right)+\tan ^{-1}\left(\frac{e^{2 \eta \rho}\left(2+e^{4 \eta \rho}-32 \eta^{3}(t+c)+4 \eta \rho\right)}{1+4 \eta e^{4 \eta \rho}\left(8 \eta^{2}(t+c)-\rho\right)}\right)\right)$

where $\rho=x-4 \eta^{2} t$. We have represented this solution for a set of parameter values in Fig. 4 .

\section{Auto-Bäcklund transformation for $\mathrm{KdV}$ equation}

Consider the KdV equation

$q_{t}+6 q_{x}+6 q q_{x}+q_{x x x}=0$. 


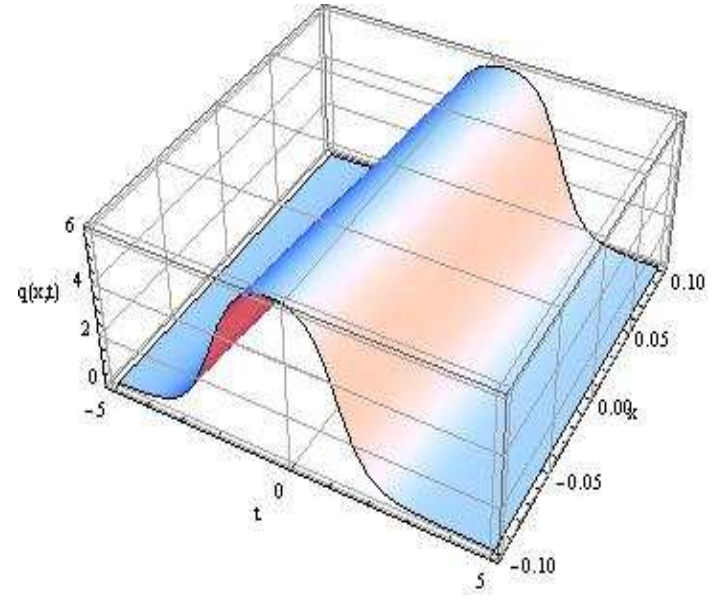

Figure 4: (a)

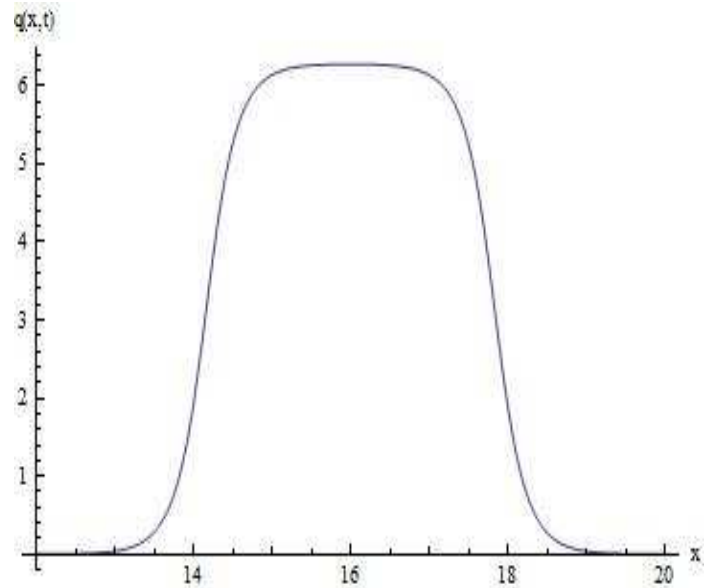

(b)

The double kink of the solution (58) for different values $\eta=1$ and $c=1$. (a)in the 3D and (b) in the plane at $t=1$.

According the idea of improved HB [9-10], we seek for ABT [23-24] of equation (59), when balancing $q q_{x}$ with $q_{x x x}$ then gives $N=2$. therefore, we may choose

$q=\frac{\partial^{2} f(\omega)}{\partial x^{2}}=f^{\prime \prime}(\omega) \omega_{x}^{2}+f^{\prime}(\omega) \omega_{x x}+u_{0}$,

where $u_{0}(x, t)$ is a solution of equation(59). Substituting equation (60) into equation (56), we obtain

$\left[6 f^{\prime \prime} f^{\prime \prime \prime}+f^{(5)}\right]\left(\omega_{x}\right)^{5}+\left[\omega_{x x t}+6 \omega_{x x x}+6 \omega_{x x x} u_{0}+6 u_{0 x} \omega_{x x}+\omega_{x x x x x}\right] f^{\prime}+\left[2 \omega_{x} \omega_{x t}+\omega_{t} \omega_{x x}+18 \omega_{x} \omega_{x x}+6 \omega_{x}^{2} u_{0 x}\right.$

$\left.+18 \omega_{x} \omega_{x x} u_{0}+5 \omega_{x} \omega_{x x x x}+10 \omega_{x x} \omega_{x x x}\right] f^{\prime \prime}+\left[\omega_{t} \omega_{x}^{2}+6 \omega_{x}^{3}+6 \omega_{x}^{3} u_{0}+10 \omega_{x}^{2} \omega_{x x x}+15 \omega_{x} \omega_{x x}^{2}\right] f^{\prime \prime \prime}+\left[10 \omega_{x}^{3} \omega_{x x}\right] f^{(4)}$

$+\left[6 \omega_{x x} \omega_{x x x}\right]\left(f^{\prime}\right)^{2}+\left[6 \omega_{x}^{2} \omega_{x x x}+18 \omega_{x} \omega_{x x}^{2}\right] f^{\prime} f^{\prime \prime}+\left[6 \omega_{x}^{3} \omega_{x x}\right]\left(f^{\prime \prime}\right)^{2}+\left[6 \omega_{x}^{3} \omega_{x x}\right] f^{\prime} f^{\prime \prime \prime}+\left[u_{0 t}+6 u_{0 x}+6 u_{0} u_{0 x}+u_{0 x x x}\right]=0$,

where $f^{(i)}=d^{i} f / d \omega^{i}$, the subscripts denote the partial derivatives, setting the coefficient of $\omega_{x}^{5}$ to zero yields an ordinary differential equation for $f$

$6 f^{\prime \prime} f^{\prime \prime \prime}+f^{(5)}=0$,

which admits a solution

$f=2 \ln \omega$.

According to (77), we get

$$
\left(f^{\prime \prime}\right)^{2}=-f^{(4)}, \quad f^{\prime} f^{\prime \prime \prime}=-\frac{2}{3} f^{(4)} \quad f^{\prime} f^{\prime \prime}=-f^{\prime \prime \prime} \quad\left(f^{\prime}\right)^{2}=-2 f^{\prime \prime} .
$$

By making use equation (64), equation (61) can be written as a finite series of $f^{\prime}, f^{\prime \prime}$ and $f^{\prime \prime \prime}$, then we get

$\left[\omega_{x x t}+6 \omega_{x x x}+6 \omega_{x x x} u_{0}+6 u_{0 x} \omega_{x x}+\omega_{x x x x x}\right] f^{\prime}+\left[2 \omega_{x} \omega_{x t}+\omega_{t} \omega_{x x}+18 \omega_{x} \omega_{x x}+6 \omega_{x}^{2} u_{0 x}+18 \omega_{x} \omega_{x x} u_{0}+5 \omega_{x} \omega_{x x x x}-2 \omega_{x x} \omega_{x x x}\right] f^{\prime \prime}$

$+\left[\omega_{t} \omega_{x}^{2}+6 \omega_{x}^{3}+6 \omega_{x}^{3} u_{0}+4 \omega_{x}^{2} \omega_{x x x}-3 \omega_{x} \omega_{x x}^{2}\right] f^{\prime \prime \prime}=q_{t}+6 q_{x}+6 q q_{x}+q_{x x x}=0$,

Decomposing the coefficient of $f^{\prime \prime \prime}$ as

$\left[\omega_{t} \omega_{x}+6 \omega_{x}^{2}+6 \omega_{x}^{2} u_{0}+4 \omega_{x} \omega_{x x x}-3 \omega_{x x}^{2}\right] \omega_{x}=A \omega_{x}$. 
Integrate the coefficient of $f^{\prime}$ with respect to $x$ once and setting the constant of integration to zero. Denote

$$
B=\omega_{x t}+6 \omega_{x x}+6 u_{0} \omega_{x x}+\omega_{x x x x} .
$$

Rewriting the coefficient of $f^{\prime \prime}$ we have

$$
\text { Coeff.of } f^{\prime \prime}=A_{x}+B \omega_{x}
$$

Substituting equations (66), (67) and (68) into equation (65) gives

$$
A \omega_{x} f^{\prime \prime \prime}+\left(A_{x}+B \omega_{x}\right) f^{\prime \prime}+B_{x} f^{\prime}=0
$$

that is

$$
\frac{2 A \omega_{x}}{\omega^{3}}-\frac{A_{x}+B \omega_{x}}{\omega^{2}}+\frac{B_{x}}{\omega}=\left(\frac{B \omega-A}{\omega^{2}}\right)_{x}=0 .
$$

Thus we have a ABT as follows

$$
q(x, t)=2[\ln \omega(x, t)]_{x}+u_{0}(x, t) .
$$

We can write the relation (70) as

$$
B w-A=\left(\omega_{x t}+6 \omega_{x x}+6 u_{0} \omega_{x x}+\omega_{x x x x}\right) \omega-\left[\omega_{t} \omega_{x}+6 \omega_{x}^{2}+6 \omega_{x}^{2} u_{0}+4 \omega_{x} \omega_{x x x}-3 \omega_{x x}^{2}\right]=0 .
$$

By equation (72)

$$
\left(D_{x} D_{t}+6\left(1+u_{0}\right) D_{x}^{2}+D_{x}^{4}\right) w \cdot w=0,
$$

where

$$
D_{x}^{m} D_{t}^{n} a . b=\left.\left(\partial_{x}-\partial_{x^{\prime}}\right)^{m}\left(\partial_{t}-\partial_{t^{\prime}}\right) a(x, t) b\left(x^{\prime}, t^{\prime}\right)\right|_{x^{\prime}=x, t^{\prime}=t} .
$$

Now setting $u_{0}(x, t)=0$ and we use the auto-BT consisted of (63) and (7) to obtain two-soliton solution of equation (59). We supposed that the solution of equation (73) in the form

$$
w(x, t)=1+\sum_{i=1}^{\infty} \varepsilon^{i} \phi_{i}
$$

where $\phi_{i}(x, t),(i=1,2,3, \ldots)$ to be determined later, $\varepsilon$ be a small parameter. Substituting (75) into (73) and collecting all terms with the same order of $\varepsilon$ equal to zero then we obtain

$$
\begin{array}{cc}
\varepsilon^{0}: & 0=0, \\
\varepsilon^{1}: & \left(D_{x} D_{t}+6 D_{x}^{2}+D_{x}^{4}\right)\left(\phi_{1} \cdot 1+1 \cdot \phi_{1}\right)=0, \\
\varepsilon^{2}: & \left(D_{x} D_{t}+6 D_{x}^{2}+D_{x}^{4}\right)\left(\phi_{2} \cdot 1+\phi_{1} \cdot \phi_{1}+1 \cdot \phi_{2}\right)=0, \\
\varepsilon^{3}: & \left(D_{x} D_{t}+6 D_{x}^{2}+D_{x}^{4}\right)\left(\phi_{3} \cdot 1+\phi_{2} \cdot \phi_{1}+\phi_{1} \cdot \phi_{2}+1 \cdot \phi_{3}\right)=0 \quad \ldots
\end{array}
$$

Note that

$$
\left(D_{x} D_{t}+6 D_{x}^{2}+D_{x}^{4}\right)\left(\phi_{1} \cdot 1\right)=\left(D_{x} D_{t}+6 D_{x}^{2}+D_{x}^{4}\right)\left(1 \cdot \phi_{1}\right)=\phi_{i x t}+6 \phi_{i x x}+\phi_{i x x x x},
$$

the linear equation $\phi_{1 x t}+6 \phi_{1 x x}+\phi_{i x x x x}$ take an exponential solution

$$
\phi(x, t)=\exp \left(\xi_{1}\right)+\exp \left(\xi_{2}\right) \quad \xi_{i}=\alpha_{i} x+\omega_{i} t+\xi_{i 0},
$$


where $\alpha_{i}, \omega_{i}$, and $\xi_{i 0},(i=1,2)$ are constants and satisfy

$$
\alpha_{i} \omega_{i}+6 \alpha_{i}^{2}+\alpha_{i}^{4}=0, \quad(i=1,2)
$$

From (50), we get

$$
2\left(\phi_{1 x t}+6 \phi_{1 x x}+\phi_{1 x x x x}\right)=-\left[D_{x} D_{t}+6 D_{x}^{2}+D_{x}^{4}\right]\left(\phi_{1} \cdot \phi_{1}\right),
$$

and

$$
\left[D_{x} D_{t}+6 D_{x}^{2}+D_{x}^{4}\right] e^{\xi_{1}} \cdot e^{\xi_{2}}=\left[\left(\omega_{2}-\omega_{1}\right)\left(\alpha_{2}-\alpha_{1}\right)+6\left(\alpha_{2}^{2}-\alpha_{1}^{2}\right)+\left(\alpha_{2}^{4}-\alpha_{1}^{4}\right)\right] e^{\xi_{1}+\xi_{2}},
$$

we have

$$
\phi_{2}(x, t)=e^{\xi_{1}+\xi_{2}+A 12}
$$

where

$$
e^{A 12}=\frac{\left(\omega_{2}-\omega_{1}\right)\left(\alpha_{2}-\alpha_{1}\right)+6\left(\alpha_{2}^{2}-\alpha_{1}^{2}\right)+\left(\alpha_{2}^{4}-\alpha_{1}^{4}\right)}{\left(\omega_{2}+\omega_{1}\right)\left(\alpha_{2}+\alpha_{1}\right)+6\left(\alpha_{2}^{2}+\alpha_{1}^{2}\right)+\left(\alpha_{2}^{4}-\alpha_{1}^{4}\right)}
$$

and

$$
\phi_{3}(x, t)=0 \quad \text { for } \quad k \geq 3
$$

Therefore the two-soliton solution of equation (1) is obtained from

$$
u(x, y, t)=2\left[\ln \left(1+\left[e^{\xi_{1}}+e^{\xi_{2}}+e^{\xi_{1}+\xi_{2}+A_{12}}\right]\right)\right]_{x},
$$

\section{Conclusion}

In this paper, the Bäcklund transformations and a series of new exact explicit solutions of the KdV-type equations have been established. We used the inverse scattering transform to derive the Bäcklund transformations for the MKdV equation see relation (A7) (in Appendix A). The solutions include $(a)$ the solitary wave solutions, $(b)$ singular traveling solutions, $(c)$ single-soliton solutions, $(d)$ Kink and double-kink solutions. In addition to some known solutions re-deriving in a systematic way, several entirely new and more general explicit exact solitary wave solutions can also be obtained. We derive the auto-Bäcklund transformation for KdV equation (1). There exist two types, the first one is the soliton, singular solutions (see Figs 3a, 3b) and the second type is the kink and double kink (see Figs 2,4) for the MKdV equation. Finally the results of this paper are useful in the interpretation of some physical phenomena, especially in plasma physics, fluid and nonlinear optics.

\section{Appendix A}

Proof of Proposition. Application of the integrability condition $\Psi_{x t}^{\prime}=\Psi_{t x}^{\prime}$ in equations (12a), (12b) and using equation (10) shows that

$$
\frac{\partial}{\partial t}\left(\frac{\partial u^{\prime}}{\partial x}+\frac{\partial u}{\partial x}\right)=4 \eta A \sin \left(u^{\prime}-u\right)+4 \eta(B-C) \cos \left(\frac{u^{\prime}-u}{2}\right)+2 \eta(B+C)+2 \eta \cos \left(u^{\prime}-u\right),
$$

and

$$
\begin{aligned}
& \frac{\partial}{\partial x}\left(\frac{\partial u^{\prime}}{\partial t}-\frac{\partial u}{\partial t}\right)=4 A_{x} \sin \left(\frac{u^{\prime}-u}{2}\right)+2 A \cos \left(\frac{u^{\prime}-u}{2}\right)\left[-2 u_{x}+4 \eta \sin \left(\frac{u^{\prime}-u}{2}\right)\right] \\
& +2\left(B_{x}-C_{x}\right)+2\left(B_{x}+C_{x}\right) \cos \left(\frac{u^{\prime}-u}{2}\right)-(B+C) \sin \left(\frac{u^{\prime}-u}{2}\right)\left[-2 u_{x}+4 \eta \sin \left(\frac{u^{\prime}-u}{2}\right)\right],
\end{aligned}
$$

from the equations (A1) and (A2) we obtain $u_{x t}=-\frac{3}{2}\left(u_{x}\right)^{2} u_{x x}-u_{x x x x}$. Similarly, application of the integrability requirement yields $u_{x t}^{\prime}=-\frac{3}{2}\left(u_{x}^{\prime}\right)^{2} u_{x x}^{\prime}-u_{x x x x}^{\prime}$. Hence, the relations (12a) and (12b) leave (3) invariant; that is, they 
constitute an ABT of the MKdV equation.

Consider now the following scattering problem:

$$
\frac{\partial \psi_{1}}{\partial x}=\eta \psi_{1}-\frac{u_{x}}{2} \psi_{2}, \quad \frac{\partial \psi_{2}}{\partial x}=-\eta \psi_{2}+\frac{u_{x}}{2} \psi_{1},
$$

by choosing the t-dependency of the eigenfunction $\psi_{1}$ and $\psi_{2}$ to be

$$
\frac{\partial \psi_{1}}{\partial t}=A(x, t, \eta) \psi_{1}+B(x, t, \eta) \psi_{2}, \quad \frac{\partial \psi_{2}}{\partial t}=C(x, t, \eta) \eta \psi_{1}-A(x, t, \eta) \psi_{2} .
$$

Defining $\Gamma=\frac{\psi_{1}}{\psi_{2}}$ the scattering equation (A4) gives the Riccati forms

$$
\frac{\partial \Gamma}{\partial x}=2 \eta \Gamma-\frac{u_{x}}{2}-\frac{u_{x}}{2} \Gamma^{2}, \quad \frac{\partial \Gamma}{\partial t}=2 A \Gamma+B-C \Gamma^{2} .
$$

We Put

$$
\left(\frac{u^{\prime}-u}{4}\right)=\tan \Gamma
$$

Substituting from equation (A6) into equation (A5) given the relation (12), Thus. from equation (A6), the BT for the MKdV-type equation in the case of the class ZS/AKNS system take the form

$$
u^{\prime}=u+4 \tan ^{-1} \Gamma \text {. }
$$

If we choose $\Gamma=\frac{1}{\Gamma^{\prime}}$ and $u^{\prime}$ as in (A7), then $\Gamma^{\prime}$ and $u^{\prime}$ satisfy equation (A5).

\section{References}

[1] M.J. Abowitz, P.A. Clarkson, Soliton, "Nonlinear evolution equations and inverse scattering". Cambridge University Press (1991).

[2] M. Ablowitz, D. Kaup, A. Newell, H. Segur, "The inverse scattering transform-Fourier analysis for nonlinear problems", Studies in Applied Mathematics, Vol.53, (1974), pp.249-315.

[3] R. Hirota "Exact solution of the Korteweg-de Vries equation for multiple collisions of solutions", Physics Review Letters, Vol.72, (1971), pp.1192-1194.

[4] J. Weiss, M. Tabor, and G. Carnevale, The Painlevé property for partial differential equations, Journal of Mathematical Physics, vol.24, No.3, (1983), pp.522-526.

[5] J. Weiss, "The Painlevé property for partial differential equations. II: Bäcklund transformation, Lax pairs, and the Schwarzian derivative", Journal Mathematical Physics, Vol.24, (1983), pp.1405-1413.

[6] O. H. EL-Kalaawy and R. B. Aldenari,"Painlevé analysis, auto-Bäcklund transformation, and new exact solutions for Schamel and Schamel-Korteweg-de Vries-Burger equations in dust ion-acoustic waves plasma",Physics of Plasmas, Vol.21, (2014), pp. 092308-092321.

[7] O. H. EL-Kalaawy and R. B. Aldenari,"Painlevé analysis, Auto-Bäcklund transformation and new exact solutions for improved modifed KdV equation", International Journal of Applied Mathematical Research, 3 (3) (2014) pp.265-272.

[8] B. Tian, Y.T. Gao, "Truncated Painlevé expansion and a wide-ranging type of generalized variable-coefficient Kadomtsev-Petviashvili equations", Physics Letters A, Vol.209, (1995), pp.297-304.

[9] M. L. Wang,", Solitary wave solutions for variant Boussinesq equations" Physics Letters A, Vol.199, (1995), pp.169-172.

[10] E. Fan and H. Q. Zhang," New exact solutions to a system of coupled KdV equations", Physics Letters A, Vol.245, (1998), pp.389-392.

[11] O. H. EL-Kalaawy,"Exact soliton solutions for some nonlinear partial differential equations" Chaos, Solitons \& Fractals, Vol.14, (2002), pp.547-552. 
[12] W. Malfliet, "Solitary wave solutions of nonlinear wave equations", American Journal Physics, Vol.60 (1992), pp. 650-654.

[13] A.M. Wazwaz, "The tanh and the sine-cosine methods for the complex modified KdV and the generalized KdV equation", Computional Mathathematical and Application, Vol.49, (2005), pp.1101-1112.

[14] M.A. Abdou, "Further Improved F-expansion and new exact solutions for nonlinear evolution equations" Nonlinear Dynamics, Vol.52, No.3, (2008), pp.277-288.

[15] M. Wang, X. Li and J. Zhang, "The $\left(G^{\prime} / G\right)$-expansion method and traveling wave solutions of nonlinear evolution equations in mathematical physics", Physics Letters A, Vol. 372, No. 4, (2008), pp.417-423.

[16] O. H. El-Kalaawy,"Exact solitary solution of Schamel equation in plasmas with negative ions",Physics of Plasmas, Vol.18 (2011), pp.112302-112309 .

[17] Y. Pandir, Y. Gurefe, and E. Misirli,"New Exact Solutions of the Time-Fractional Nonlinear Dispersive KdV Equation", International Journal of Modeling and Optimization, Vol. 3, No. 4, (2013), pp.349-352.

[18] C. Rogers and W.E. Shadwisk," Bäcklund transformations and their applications," Academic Press, New York, (1982).

[19] M. Wadati, H. Sunukt and K. Konno, "Relationships among inverse method, Bäcklund transformation and an infinite number of conservatioq laws", Progress Theortical Physics, Vol.53, (1975), pp.419-436.

[20] A.H. Khater, M.A. Helal and O.H. El-Kalaawy, "Two new classes of exact solutions for the KdV equation via Bäcklund transformations", Chaos Solitons Fractals, Vol.8, (1997), pp.1901-1907.

[21] K. Konno, M. Wadati, "Simple derivation of Bäcklund transformation from Riccati form of inverse method", Progress. Theoret. Phys., Vol.53, (1975), pp.1652-1655.

[22] G B Airy, In Encycllopecdia metropolotana 5 (241 London) (1845).

[23] Liu Chun-Ping and Zhou Ling," A new auto-Bäacklund transformation and two-soliton solution for (3+1)-dimensional Jimbo-Miwa equation" Communications in Theoretical Physics, Vol.55, (2011), pp.213-216.

[24] Qin Yi, Gao Yi-Tian, Yu Xin and Meng Gao-Qing,"Bell polynomial approach and N-soliton solutions for a coupled KdV-mKdV system " Communications in Theoretical Physics, Vol.58, No.1, (2012) pp.73-77. 